\title{
EFICIENCIA DE CONVERSIÓN DE HÍBRIDOS EXPERIMENTALES PARA LA PRODUCCIÓN DE POLLOS CAMPEROS
}

\author{
DotTAVIO, A. M. ${ }^{1,3}$; LIBRERA, J. E. ${ }^{1}$; ROMERA, B. M. ${ }^{1}$; \\ FONT, M. T. ${ }^{2,3} ;$ DI MASSO, R.J. ${ }^{1,2,3}$
}

\begin{abstract}
RESUMEN
En la avicultura comercial la alimentación representa aproximadamente el $80 \%$ del costo de producción. La eficiencia de conversión es un descriptor biológica y económicamente útil de la relación entre la tasa de crecimiento y el consumo de alimento. El pollo Campero INTA es un ave de crecimiento más lento que las líneas comerciales de pollos parrilleros, y la caracterización del patrón de crecimiento de seis híbridos experimentales alternativos al híbrido tradicional mostró, en alguno de ellos, una reducción en la tasa de maduración sin efectos significativos sobre el tamaño maduro, lo que se tradujo en un aumento de la edad a la faena. El impacto de la modificación del patrón de crecimiento sobre la eficiencia de conversión se evaluó en los seis híbridos experimentales. Los machos del híbrido Tradicional II tuvieron similar aumento medio diario de peso y consumo voluntario de alimento que el híbrido Campero INTA y no se diferenciaron de éste en la eficiencia de conversión del alimento, mientras que las hembras fueron menos eficientes. En ambos sexos, las combinaciones híbridas restantes mostraron similar aumento diario de peso, menor consumo diario de alimento y mayor eficiencia (menor relación de conversión) que el genotipo de referencia Campero INTA pero presentaron menor peso corporal promedio que este último a la edad fija analizada. La mejor eficiencia biológica de estos híbridos alternativos derivada del menor peso corporal mantenido en el lapso prefijado no se tradujo en una mejor eficiencia económica dado que estas aves se faenaron a mayor edad.
\end{abstract}

Palabras clave: consumo de alimento, ganancia de peso, eficiencia, híbridos, pollo campero.

\section{SUMMARY}

\section{Feed efficiency in experimental hybrids for free-range broilers produc- tion.}

Six hybrids produced as alternatives to Campero INTA free-range birds showed a reduction in maturing rate without significant effects on asymptotic body weight in four of them. These birds needed more time to reach the target slaughter weight and were sacrificed at an older age. The

1. Cátedra de Genética. Facultad de Ciencias Veterinarias. Ovidio Lagos y Ruta 33, 2170 Casilda.

2. Instituto de Genética Experimental. Facultad de Ciencias Médicas. Santa Fe 3100, 2000 Rosario.

3. Carrera del Investigador Científico CIC-UNR. Universidad Nacional de Rosario. República Argentina. Manuscrito recibido el 2 de julio de 2007 y aceptado para su publicación el 20 de mayo de 2008. 
objective of this study was to characterize the effect of this change in growth pattern on feed efficiency. Males of the Tradicional II combination showed similar weight gain and feed intake when compared to Campero INTA birds, without differences in feed efficiency meanwhile females were less efficient. All other combinations showed, in both sexes, similar weight gains, lower feed intakes and higher feed efficiencies than Campero INTA birds but were lighter than the control genotype. The higher biological efficiency was not accompanied by a better economic efficiency because the target slaughter weight was achieved at an older age.

Key words: feed intake, growth rate, feed efficiency, hybrids, free-range broilers. 